\title{
Bridging to transplantability with a ventricular assist device
}

\author{
E. V. Potapov, MD, Y. Weng, MD, PhD, M. Jurmann, MD, H. Lehmkuhl, MD, and R. Hetzer, MD, PhD, Berlin, Germany
}

$\mathrm{T}$ he implantation of a ventricular assist device (VAD) is an accepted option in patients with end-stage heart failure. However, the durability of these devices is limited to a number of years, and complications still limit outcome. In children and young adults with end-stage heart failure and contraindications for heart transplantation, there are thus no long-term options available.

\section{Clinical Summary}

A 15-year-old girl had osteosarcoma of the left femur, which was radically operated on in December 2001. The patient received postoperative chemotherapy with doxorubicin and cisplatin for 5 months. In May 2002, cardiomyopathy developed, subsequently leading to biventricular heart failure. The patient's condition deteriorated rapidly, with lung edema and signs of multiorgan dysfunction necessitating inotropic support and ventilation. In June 2002, the patient was transferred from the Netherlands to Berlin and immediately supported with an extracorporeal biventricular assist device (Berlin Heart, Berlin, Germany). The postoperative course was uneventful. One month later, spongioplasty restored the integrity and mechanical function of the femur. The girl was discharged home in September 2002 and went back to school. In June 2003, 1 year and 7 months after tumor removal, a computed tomographic scan showed no evidence of tumor recurrence or metastatic processes. The patient was listed for heart transplantation and received a donor heart after 420 days on support, 10 days after her 17th birthday. Again, the postoperative course was uneventful. Almost 2 years after heart transplantation, despite triple immunosuppression protocol, the malignancy remains in remission.

\section{Discussion}

A few cases have been reported of urgent heart transplantation after treatment of osteosarcoma (for example, at 16 months, ${ }^{1} 2$

From the Department of Cardiothoracic and Vascular Surgery, Deutsches Herzzentrum, Berlin, Germany.

Received for publication April 14, 2005; accepted for publication April 28, 2005.

Address for reprints: Evgenij V. Potapov, MD, Deutsches Herzzentrum Berlin, Augustenburger Platz 1, 13353 Berlin, Germany (E-mail: potapov@dhzb.de).

J Thorac Cardiovasc Surg 2005;130:930

$0022-5223 / \$ 30.00$

Copyright $\odot 2005$ by The American Association for Thoracic Surgery

doi:10.1016/j.jtcvs.2005.04.018 years and 5 months, ${ }^{2}$ and 10 years $^{3}$ ) because of slow progression of chemotherapy-induced cardiomyopathy. In our case of rapid deterioration, however, VAD implantation was the only means of saving the patient's life and gaining time. This case opens up new horizons for VAD implantation as a "bridge to transplantability" for patients with contraindications for heart transplantation, in addition to the established indications of direct bridging to heart transplantation or myocardial recovery or as a definitive therapy. In some patients with fixed pulmonary hypertension precluding heart transplantation, long-term support with a left VAD leads to a significant and sustained decrease in pulmonary vascular resistance, allowing successful heart transplantation after 3 to 6 months with the left VAD. ${ }^{4}$ During long-term support with a VAD, infective, thromboembolic, or bleeding complications may arise; it is therefore an individual decision as to how long the patient should be mechanically supported before the necessary freedom from tumor recurrence can be postulated. In this case, freedom from tumor for 1 year and 7 months allowed heart transplantation to be performed with subsequent immunosuppression and without tumor recurrence to date, 1 year and 9 months later. Despite the promising long-term prognosis, ${ }^{1,3,5}$ close posttransplantation follow-up focusing on tumor recurrence will remain necessary. In our case, long-term VAD support allowed the patient to be listed for heart transplantation after she had reached a status of transplantability. In such cases, as well as for patients with elevated pulmonary vascular resistance, bridge to transplantability may represent a new concept for long-term VAD support.

We thank Anne M. Gale, Editor in the Life Sciences, for editorial assistance.

\section{References}

1. McManus RP, O'Hair DP. Pediatric heart transplantation for doxorubicin-induced cardiomyopathy. J Heart Lung Transplant. 1992;11: 375-6.

2. Arico M, Nespoli L, Pedroni E, Bonetti F, Vigano M, Burgio GR. Heart transplantation in a child with doxorubicin-induced cardiomyopathy. N Engl J Med. 1988;319:1353.

3. Hinkamp T, Sullivan H, Bakhos M, Grieco J, Pifarré R. Orthotopic cardiac transplantation in two patients with previous malignancy. Ann Thorac Surg. 1991;51:1004-6.

4. Martin J, Siegenthaler MP, Friesewinkel O, Fader T, van de Loo A, Trummer G, et al. Implantable left ventricular assist device for treatment of pulmonary hypertension in candidates for orthotopic heart transplantation-a preliminary study. Eur J Cardiothorac Surg. 2004; 25:971-7.

5. Arico M, Pedroni E, Nespoli L, Vigano M, Porta F, Burgio GR. Long term survival after heart transplantation for doxorubicin induced cardiomyopathy. Arch Dis Child. 1991;66:985-6. 